Running head: CULTURAL RISK MARKERS FOR MALE PERPETRATION OF IPV

\title{
A Meta-Analysis of Cross Cultural Risk Markers for Intimate Partner Violence
}

Mallory, A., Deitz, S., Barros- Gomez, P., Dharnidharka, P., Cafferky, B., Van, K., Stith, S.

\begin{abstract}
The majority of IPV research has originated from western countries and neglected to examine cultural influences. We meta-analyzed the strength of various well-established risk markers (demographic, individual-level and relational) for male-perpetrated IPV across different cultures. Using Hofstede's (2010) individualism scale, we grouped studies from countries into individualist and collectivist categories, and then accounted for the influence of the large number of U.S.-based studies, by creating 3 groups: U.S., Individualistic, and Collectivist. Risk markers across collectivist and U.S. groups had similar effect sizes across while risk markers in the U.S. had larger effect sizes than those found in the international individualist countries. Our findings suggest that if a comprehensive understanding of IPV perpetration is to be achieved, cultural context cannot be ignored.
\end{abstract}


A Meta-Analysis of Cross Cultural Risk Markers for Intimate Partner Violence Intimate partner violence (IPV) is a serious public health concern around the world (Garcia-Moreno, Pallitto, Devries, Stockl, Watts, \& Abrahams, 2013). About 30\% of women worldwide have experienced physical and/or sexual violence by intimate partners at some point in their lives (World Health Organization, García-Moreno, Pallitto Devries, Stockl, Watts, \& Abrahams, 2013). About $38 \%$ of female homicides in the world are committed by intimate partners. Despite the ubiquity of male-perpetrated IPV in heterosexual relationships around the world, research on the subject has been predominantly located in the West (Jeyaseelan, Sadowski, Kumar, Hassan, Ramiro, \& Vizcarra, 2004; Krahé, Bieneck, \& Möller, 2005). Thus, most of our knowledge about IPV risk markers is derived from populations in western countries. Further, although there are several meta-analytic studies of risk markers for IPV in the U.S., few studies have systematically reviewed the knowledge generated on IPV outside the U.S. Accordingly, this meta-analytic study, examines and compares demographic, individual, familyof-origin and relational risk markers for male-perpetrated IPV in western and non-western countries.

Culture - an often neglected dimension in IPV research - is fundamental in shaping a person's worldview, views of self, and all social phenomena (Yoshioka \& Chan, 2005). As we seek to increase our understanding of IPV around the world, it is important to consider cultural factors and values underlying IPV perpetration. Although research on risk markers for male perpetrated IPV is growing around the world (García-Moreno et. al., 2013), to our knowledge there are no meta-analyses examining the affect cultural influences have on the link between risk markers and IPV. The goal of this meta-analysis was to examine the relative strength of risk markers for male perpetrated IPV across different countries. Using the individualism dimension 
from Hofstede's Dimensional Model of Culture, we grouped countries into 'individualistic' and 'collectivist' categories (Hofstede, Hofstede, and Minkov, 2010). Because the majority of the studies categorized as individualist were conducted in the U.S., we compared risk markers across three groups: studies conducted in the U.S., studies conducted in non-U.S. individualist countries, and studies conducted in collectivist countries. This study makes an important contribution to the field of IPV research by examining how broad cultural differences may affect risk markers linked to male-perpetrated physical IPV.

\section{Background}

\section{Theoretical Review}

Hofstede's Dimensional Model of Culture has been used to understand variation in cultural values across different countries (Hofstede, 2011). This model emerged from Hofstede's values survey completed by IBM employees from 50 countries in the 1970's. Initial correlational analyses revealed that there were significant stable differences between mean survey item scores at the country level. Hofstede replicated these findings with 400 non-IBM management trainees from 30 countries which showed a similar pattern of differences between countries. A factor analysis of the data revealed four dimensions of national culture: Power - Distance, Uncertainty Avoidance, Individualism-Collectivism and Masculinity-Femininity (Hofstede, 1984).

In the present paper, we focus on how the cultural dimensions of individualism and collectivism are associated with risk markers for IPV perpetration. Hofstede (2011) describes individualism and collectivism as societal level characteristics. Hofstede broadly describes individualist societies as those that have "loose ties between individuals" (p. 11) and people look after themselves and their immediate family. Collectivism is broadly described as cultures where 
“...people from birth onward are integrated into strong cohesive groups, often extended families...that continue protecting them in exchange for unquestioning loyalty" (p.11).

Hofstede, et al, (2011) suggest that individualist cultures tend to privilege "I" consciousness, right to privacy, speaking one's mind, personal opinion and tasks over relationships. "Others" are classified as individuals and deviating from norms leads to feelings of guilt (Hofstede et al., 2011). Collectivist societies, on the other hand, emphasize "we" consciousness, belonging, harmony and relationships over tasks. "Others" are classified as in- or out-group, opinions are determined by the in-group and deviating from norms leads to feelings of shame (Hofstede et al., 2011). Using Hofstede's individualism-collectivism scale, countries are scored on a continuum from 0 to 100: with 100 representing strongly individualist countries, 50 as the midpoint between individualist and collectivist and zero strongly collectivist countries. Developed and western countries tend to be higher on individualism, while lesser developed and eastern countries tend to be lower on the individualism index, and Japan occupies a position near the center. Hofstede's continuum could be helpful to broadly distinguish between individualist and collectivist cultures, while still recognizing the presence of heterogeneity within individualist cultures and within collectivist cultures.

\section{Review of Risk Markers of IPV}

Research in developing countries has predominantly focused on a limited number of risk markers linked to IPV in the U.S. such as socio-demographic factors, alcohol use and intergenerational transmission of abuse (Chan, 2004; Eisikovits, Winstok \& Fishman, 2004; Koenig, Ahmed, Hossain, \& Mozumder, 2003). Although research on female IPV perpetration has grown dramatically in the U.S., research on female IPV perpetration internationally is limited. Therefore, the current meta-analytic review includes only studies of male perpetrated 
IPV and only includes risk markers which had at least three effect sizes in collectivist countries, individualist countries, and the U.S. Below, we review evidence for risk markers included for all three groups and offer hypotheses regarding each type of risk marker. The risk markers are categorized as demographic, individual, family of origin (FOO), and relational.

\section{Demographic Risk Markers.}

Age, education, income and employment status were included as demographic risk markers in this study. Research in the U.S. has established strong links between IPV and these demographic factors (Capaldi, Knoble, Shortt, \& Kim, 2012). Younger age individuals have consistently been found to have a higher probability of being violent in intimate relationships (Capaldi et al., 2012). Further, low income and unemployment are associated with higher risk for IPV and are generally found to be more reliable risk markers than educational level (Capaldi et al., 2012). Research outside the U.S. shows similar trends For instance, in an Israeli study of men and women in violent versus nonviolent relationships, violent men were more likely to be younger, less educated, and more likely to be unemployed than men who were not violent (Eisikovits et al., 2004). However, in one study done in China, husbands' younger age, lower education level and lower income were not significantly related to increased perpetration of physical IPV (Hou, Yu, Ting, Sze, \& Fang, 2011). There were similar findings in a comparative study in India, with no significant difference indicated between husbands' age and husbands' employment for women in physically violent relationships compared to women not in violent relationships (Krishnan, Rocca, Hubbard, Subbiah, Edmeades, Padian, 2010). Thus, it is not clear how these demographic risk markers might be related to culture. However, we hypothesize that because family may play a more central role in collectivist countries than in individualist 
countries, the risk markers of age, education, income, and employment status may be less predictive of IPV in collectivist countries than in individualist countries.

\section{Individual Risk Markers.}

Alcohol and drug use and abuse were considered individual-level risk markers for IPV in the present study. The link between alcohol use and male IPV has long been established in the U.S. with effect sizes ranging from small-to-moderate (Author, et. al., in press). Alcohol use has been similarly linked with IPV in studies outside the U.S. For instance, in a study of IPV risk markers in Chile, India, Egypt and Philippines, Jeyaseelan and colleagues (2004) found that alcohol abuse was a strong risk marker for male perpetrated IPV in all four countries. Drug abuse, on the other hand, has been investigated to a lesser extent within and outside the U.S. (Capaldi et al., 2012).

In a nationally representative study conducted in the U.S., researchers found that both alcohol and specific substances (i.e. sedatives, cocaine, cannabis, and nicotine) were significantly related to physical IPV with sedatives, cocaine, and cannabis having larger odds of physical IPV. Outside of the U.S. a few studies examining the link between drug abuse and physical IPV have been conducted. One study from Nigeria found that a partner ever abusing a substance was a related to female participants' experiencing lifetime IPV (Owoaje \& OlaOlorun, 2012). However, the number of partners that abused substances was very small $(n=12)$. A larger study conducted in Brazil found that respondent's partner's use of drugs was associated with IPV (Vieira Perdona, dos Santos, 2011). One limitation of both of these studies was that drug abuse was a dichotomous yes/no question which doesn't allow for a nuanced understanding of the relationship between drug use and physical IPV (e.g. partners may have abuse drugs once or daily). U.S. studies suggest that drug use may be more strongly associated with the risk for IPV as compared to alcohol use (Capaldi et al., 2012). No research has examined whether or not the 
impact of problems with substance use on IPV perpetration might be related to culture, therefore we do not propose a directional hypothesis regarding cultural differences on the impact of alcohol and drug use on IPV.

\section{Family of Origin Risk Markers.}

Witnessing inter-parental violence and experiencing child physical abuse - two common measures of family of origin violence-were included in this study. U.S. meta-analyses revealed small to moderate effect sizes for both these risk markers (Author, 2015; Author, 2000). Although there are no meta-analytic or systematic reviews of the direction and strength of the association between family of origin violence and adult IPV available for countries outside the U.S., individual studies conducted internationally indicate a similar relationship. For instance, a population-based household survey of IPV in India found that women who reported witnessing inter-parental violence and experiencing child abuse were at a greater risk for victimization (Jeyaseelan et al., 2004). Further, in a sample of Pakistani men, both being beaten as a child and witnessing a mother being beaten as a child were related to a greater likelihood of men perpetrating physical IPV against their wife - being beaten as a child was also the strongest factor included in the study (Fikree, Razzak, \& Durocher, 2005). Similarly, husbands' witnessing their mother being hit as a child was also the strongest factor for women's victimization in study conducted in Indonesia (Hayati, Hogberg, Hakimi, Ellsberg, \& Emmelin, 2011). While it is not known how family of origin risk markers differ by country, we hypothesize that these factors may be stronger risk markers in collectivist countries than in individualist countries because of the stronger connection between family of origin and current relationships in collectivist countries.

\section{Relationship Risk Markers.}


Emotional abuse, power and control, and relationship satisfaction were examined as relationship-level risk markers. Emotional or psychological abuse has been defined as "acts of recurring criticism and/or verbal aggression toward a partner, and/or acts of isolation and domination of a partner" (O'Leary 1999, p. 19). Internationally and in the U.S., although it has been difficult to establish reliable prevalence rates of psychological abuse due to definitional and measurement issues (García-Moreno et al., 2013; O’Leary, 1999), it has been established in Western literature that emotional abuse co-occurs with physical violence and is predictive of physical IPV (Murphy \& O'Leary, 1989; Schumacher \& Leonard, 2005). A meta-analysis of risk markers for IPV revealed a large effect size for the association between emotional abuse and male-to-female IPV highlighting its importance in the study of IPV (Author, 2004).

Internationally, lifetime prevalence rates of psychological or emotional abuse ranged from $13.5 \%$ to $68 \%$ in Asia, $26 \%$ to $82.6 \%$ in the Middle East, $18.9 \%$ to $30.7 \%$ in Africa, from $11.5 \%$ to 80.2\% in Latin America and the Caribbean and from $19 \%$ to $61.5 \%$ in Europe (EsquivelSantoveña, Lambert \& Hamel, 2013). Given the high rates of prevalence of emotional abuse worldwide and its strong relationship with physical IPV in western countries, it is an important risk marker to examine within and outside the U.S.

The use of power and control in an intimate relationship is a risk marker closely tied to emotional abuse and IPV (Langhinrichsen-Rohling, 2010). Although power and control has received much scholarly attention, its link with IPV is not as clear as emotional abuse. For instance, a large case-control study conducted from 1994 to 2004 with urban women from eleven U.S. cities found that while controlling behaviors were not significant risk markers for IPV, it was significantly associated with the risk for injury (Walton-Moss, Manganello, Frye, \& Campbell, 2005). Control or controlling behaviors are also commonly assessed as risk markers 
in the literature outside the U.S. Internationally, individual studies have indicated that controlling behaviors significantly increase the likelihood of IPV (Vieira, Perdona, \& Santos, 2011; Heaton \& Forste, 2008). Therefore, it is evident that although the relationship between power and control and IPV may not be clear, it is an important variable to examine around the world. Relationship satisfaction is among the most commonly investigated relationship markers for IPV. Evidence suggests that low levels of relationship satisfaction are closely associated with IPV and mediate the relationship between other risk markers such as alcohol abuse and IPV (Smith Slep et al., 2010; White and Chen, 2002). Author's (2004) meta-analytic review indicated that relationship satisfaction has a small effect on IPV. There has been less research examining relationship satisfaction outside of the U.S. These studies have largely found relationship satisfaction to be a significant, negatively associated, and relatively small risk marker for IPV in adult relationships (Burrus \& Cobb, 2011, Hadas et al., 2008; Jose, 2010; O'Leary et al., 2008). Given the importance of relationship satisfaction as a risk marker and the simultaneously small number of studies conducted outside of the U.S., integrating findings from across the world will help elucidate the role culture plays in informing our understanding of relationship satisfaction as a risk marker for IPV around the world. Although we hypothesize that power and control may be a stronger risk marker in collectivist countries than in individualist countries because of the stronger influence of patriarchy, we hypothesize that relationship satisfaction may be more strongly related to IPV in individualist countries than in collectivist countries because of the importance of individual choice in mate selection in individualist countries..

In summary, there is a need to examine the relative strength and associations between risk markers linked with IPV in the U.S. with counties around the world from a cultural lens in 
order to develop evidence-based and culturally informed intervention and prevention programs. The present study uses a meta-analytic framework to integrate knowledge generated about IPV risk markers using studies within and outside the U.S.

\section{Method}

\section{Search Strategies}

Selection and identification of appropriate studies occurred in multiple phases. We first reviewed 509 studies from 1980 to 2000 , which were considered in a previous meta-analysis of IPV risk markers (Author). These studies were obtained from computer database searches (ERIC, Sociological Abstracts, Medline, PsychLit, Social Sciences Abstracts, and Social Sciences Citations Index). The key words from the previous meta-analysis were adapted and used in this present study (see Author, 2004) for a detailed list of terms). The references from each included study were hand-picked for additional studies to be potential included in the analysis.

Next, several databases (Web of Science, PROQUEST, and MEDLINE) were searched for all studies (including dissertations and theses) published from January 2001 to December 2012 with key words related to couple (intimate partner, marital, spouse, husband, wife, or same-sex partner), partner aggression (abuse, aggression, domestic violence, batter, maltreatment, or violence), and risk markers (risk, factor, predictor, pathway, or correlate).

We then employed a variety of hand-picking methods to assure higher levels of study inclusion. We searched through all available issues of journals which focused on family violence (Violence and Victims, Journal of Family Violence, Journal of Interpersonal Violence, Partner Abuse, Journal of Aggression, Maltreatment, and Trauma, Violence Against Women, and Psychology of Violence). We searched abstracts from national conference presentations (American Association for Marriage and Family Therapy, National Council on Family Relations, 
National Association for Social Work, and International Family Violence and Child Victimization Research Conference) to contact presenters and inquired about their unpublished findings. We also contacted prominent authors in the field of IPV about additional unpublished papers/presentations. Finally, we hand-picked the reference lists from twelve focal IPV metaanalyses/comprehensive reviews and hand-picked the reference lists from any included studies published after 2009. Whenever a study reported insufficient data to calculate a usable effect size, we emailed the first author to request additional information (11\% positive response rate).

\section{Inclusion Criteria}

The inclusion of studies in the meta-analysis was based on several criteria and had two stages: selection and screening (Author et. al., 2004). Researchers read titles and abstracts of potential studies in order to select them for closer screening. If the screened studies adhered to our inclusion and exclusion criteria, they were included in the meta-analysis. Included studies had to meet the following criteria: (a) published in English, (b) include statistics sufficient for calculating at least one bivariate effect size, (c) have an adult sample that was married or cohabitating, and (d) use physical IPV as the outcome. However, studies were excluded if: (e) their samples were solely comprised of a special population (such as those with traumatic brain injuries), (f) they focused on dating violence or university samples (because dating violence is considered to be a different area of research), (g) they did not specifically report on physical IPV as an outcome (i.e. they combined physical, psychological, and sexual IPV as one outcome), (h) focused on intimate partner homicide, or (i) did not differentiate results by perpetration and/or victimization.

\section{Coding Procedures}


We followed recommended meta-analytic coding procedures (Card, 2012; Hunter \& Schmidt, 2004). A research coding team was trained by project leaders, who were two Ph.D. students and one faculty member, to use the 37-item code sheet to capture sample, bibliographical, and study information from each included study. When coders were unable to arrive at an agreement about the correct information to be included while cross coding, the discrepancy was noted and they consulted with a project leader to arrive at a more accurate understanding of the data (Hawkins, Blanchard, Baldwin, \& Fawcett, 2008). Of the studies included in this analysis, $75 \%$ were cross-coded by two separate researchers. Coders experienced a $96 \%$ agreement rate.

\section{Grouping by Culture and Risk Marker}

We identified the countries from which each included study's sample was drawn, and then used each country's ranking on Hofstede et al.'s (2010) individualism scale to code whether the sample was from a collectivist or individualist culture. Countries were coded on a scale from 1-100; a score of 1-49 was considered collectivist and those above 50 and above were considered as individualistic (Hofstede et al., 2010). For countries not included in Hofstede's individualism scale, a literature search was conducted to find evidence supporting grouping a country into one of the categories. Specifically, Ukraine was determined to be an individualist country and Bolivia, Cambodia, Myanmar, Palestine, Nicaragua, Puerto Rico, Rwanda, Togo, and Uganda were considered collectivist based on the extant literature.

After grouping the studies by collectivist versus individualist cultures, we assessed the frequency of various risk markers reported in these studies. If there were at least 3 usable effect sizes found in the individualist and collectivist groups then that risk marker was included in this meta-analysis. The majority of risk markers were reported by male perpetrators but we also 
included effect sizes from studies female participants reporting on a male partner's violence.

This exception was made for studies where participants were women but they reported on a risk marker for the husband. For example, a female participant may have reported her partner's age or that her partner witnessed his parents hitting each other and that he was physically violent to her. Since the risk marker is about the male partner and him perpetrating physical IPV against a female partner, these studies were included. No studies were included that only had a female reporting on her own risk markers and male-to-female physical IPV. It was also important to include studies where female partners reported on risk markers for male perpetration because many studies outside of the U.S. did not have couple level data or men self-reporting on their perpetration and we would have excluded a number of useful studies using this criteria.

Although the U.S. is considered highly individualist (a score of 91/100 on Hofstede et al.'s scale; 2010), we separated the U.S. samples from the international individualist samples for several reasons. The majority of the literature on IPV originates from the U.S. and most of the instruments used to measure risk markers were developed in the U.S. Likewise, we had substantially more effect sizes contributed from U.S. samples compared to individual international samples and we did not want the mean effect size from the individualist group to be skewed toward the U.S. Furthermore, splitting the individualist group into two groups allows for specific exploration of significant differences between three different groups (international individualist studies, the collectivist studies, and U.S. studies). Based on Hofstede's spectrum, splitting the individualist group into two groups may be a better theoretical fit as it accounts for potential heterogeneity within the individualist countries.

We recognize that multiple differences exist between research participants in all countries and cultures, that there is a wide spectrum of variation within our grouping factor of 
collectivist/individualistic, and that there is no perfect way to categorize vibrantly divergent cultures into simple groups. However, an enhanced understanding of the similarities and differences based on each country's collectivist/individualist orientation can enhance our understanding of how broad systemic collectivist/individualist ideals may impact the strength of the link between prevalent risk markers and male perpetration of IPV.

\section{Statistical Methods}

Comprehensive Meta-Analysis (Borenstein, Hedges, Higgins, \& Rothstein, 2005) was used to analyze the link between risk markers and male IPV perpetration while accounting for the three cultural groups. We used a random-effects model to yield aggregate effect sizes and account for within-study and between-study variances which allows for more generalized inferences beyond the included studies of the meta-analysis (Card, 2012) A random-effects model was also used in combining across cultural subgroups in order to estimate the betweensubgroup differences, allowing for generalizable inferences beyond the included studies included in the analysis.

\section{Results}

\section{Included Samples}

Using the selection criteria outlined above, we included 49 studies from collectivist samples, 31 from individualist samples outside the U.S., and 210 from the U.S., for a total of 291 studies representing a combined sample size of 225,822 and yielding 779 independent effect sizes $(k)$. Of these effect sizes, 107 were from collectivist countries, 92 from international individualist countries, and 580 from the U.S. A list of international individualist and collectivist countries and the number of studies from each country is included in the notes of Table 2 .

\section{Publication Bias}


Meta-analyses suffer from the "file-drawer problem", in that units of measurement may be limited to results reported in published studies, which typically report significant findings (Hunter \& Schmidt, 2004). Although we included a number of unpublished studies, there are undoubtedly some unpublished studies we could not obtain. We conducted three analyses to evaluate the possible effect that publication bias could have on our mean effect sizes using the trim and fill test (Duval \& Tweedie, 2000), fail safe N (Rosenthal, 1979), and Orwin's fail safe N (Orwin, 1983). The results of all three publication bias tests indicated this meta-analysis is robust against publication bias (see Table 1).

Table 1

Duval and Tweedie's Trim and Fill (Random Effects), Classic Fail-Safe N, and Orwin's FailSafe N Tests for Risk markers Associated with Male Perpetration of IPV

\begin{tabular}{|c|c|c|c|c|}
\hline & & Trim and Fill & Classic & Orwin's Fail-Safe N \\
\hline Risk Marker & $\mathrm{K}$ & Imputed Studies & Fail-Safe N & r to .10 \\
\hline
\end{tabular}

Demographic Risk Markers

$\begin{array}{lcccc}\text { Age } & 79 & 9 & 5376 & 93 \\ \text { Education } & 71 & 2 & 6279 & 883 \\ \text { Employment } & 37 & 0 & 641 & - \\ \text { Income } & 55 & 26 & 6514 & 519\end{array}$

Individual Risk Markers

$\begin{array}{lcccc}\text { Alcohol Abuse } & 163 & 14 & 9324 & 2961 \\ \text { Drug Abuse } & 63 & 8 & 1808 & 1643\end{array}$

\section{Family of Origin Risk Makers}

Child Abuse in FOO

Witnessing Parental IPV
51

59
11

14
6288 923

1583 


\section{Relationship Risk Markers}

\begin{tabular}{lcccc} 
Control & 30 & 6 & 4214 & 1247 \\
Emotional Abuse & 97 & 11 & 832 & 4315 \\
Relationship Satisfaction & 72 & 5 & 9218 & 1738 \\
\hline Note: We did not calculate Orwin's Fail-Safe N Nor Employment because the effect sizes were \\
already small.
\end{tabular}

\section{Types of Violence}

Previous research (Johnson, Leonard, Xu, 2014) has shown that IPV can be grouped into two types of violence, i.e., situational violence and intimate terrorism. Few studies have made use of these different types of violence despite research showing differing experiences of violence and outcomes based on type of violence. Guided by the notion that clinical samples are more likely to be characterized by intimate terrorism and community samples by situational violence (Johnson \& Cares, 2004), we attempted to test for these two types of violence by comparing the strength of risk markers taken from clinical versus community populations. However, we found no significant differences between the three cultural groups when comparing clinical versus community sample effect sizes for these risk markers.

\section{Analysis Plan}

We first conducted a three group analysis between the U.S., international individualist countries, and collectivist countries to test if there were any significant group differences. Next, two group analyses were computed, by comparing international individualist countries with the U.S., then the U.S. with collectivist countries, and finally the international individualist countries with collectivist countries. The results of these tests are reported in this same sequence.

\section{Collectivist Countries, International Individualist Countries, and United States.}


We tested eleven risk markers for male perpetrated IPV across three different groups (U.S., international individualistic, and collectivist groups; see Table 2). Seven of these risk markers did not differ between these three cultural groups, but four risk markers were found to be significantly different between the three cultural groups: age $\left(Q^{b}(2)=6.39, p<.05\right)$, witnessing parental IPV $\left(Q^{b}(2)=11.82, p<.01\right)$, emotional abuse $\left(Q^{b}(2)=10.06, p<.01\right)$, and relationship satisfaction $\left(Q^{b}(2)=8.55, p<.01\right)$, Thus, one demographic, individual, and family of origin risk marker differed significantly between the three groups and two relationship risk markers differed significantly between the three groups.

Young age was a larger risk marker for the U.S. group $(r=-.14, p<.001)$, followed by the international individualistic countries $(r=-.08, n s)$, and collectivist countries $(r=-.05, \mathrm{p}$ $<.01)$. As hypothesized witnessing parental IPV was a larger risk marker in collectivist countries $(r=.38, p<.001)$, followed by the U.S. $(r=.24, p<.001)$, and international individualistic countries $(r=.20, p<.001)$. Emotional abuse was a larger risk marker in collectivist countries $(r$ $=.63, p<.001)$, followed by the U.S. $(r=.52, p<.001)$ and international individualistic countries $(r=.40, p<.001)$. Low relationship satisfaction was a larger risk marker in the U.S. $(r=-.27, p$ $<.001)$, followed by collectivist countries $(r=-.23, p<.01)$, and international individualistic countries $(r=-.13, p<.001)$.

In summary, in the three group comparison, we found young age and low relationship satisfaction were stronger risk markers in the U.S. compared to the other groups and witnessing parental IPV and emotional abuse were stronger risk markers in collectivist countries compared to the other groups. After this three group comparison, we systematically paired each of these groups together for a more specific comparison analyses. 
Table 2

Three Group Analysis of Risk markers for Male Perpetration of IPV: Collectivist countries, International Individualist countries, and the United States

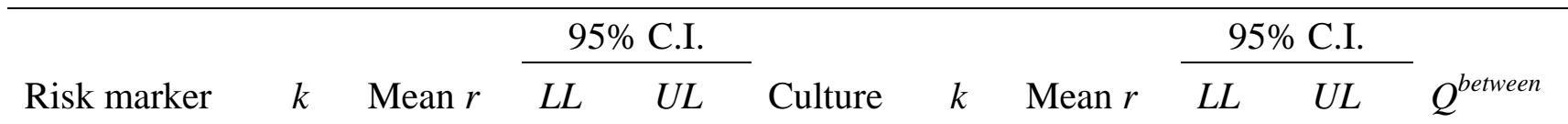

Demographic Risk Markers

\begin{tabular}{|c|c|c|c|c|c|c|c|c|c|c|}
\hline \multirow{3}{*}{ Age } & \multirow{3}{*}{79} & \multirow{3}{*}{$-0.10 * *$} & \multirow{3}{*}{-0.17} & \multirow{3}{*}{-0.02} & $\mathrm{COL}$ & 10 & -0.05 & -0.12 & -0.01 & \multirow{3}{*}{$6.39 *$} \\
\hline & & & & & IIND & 3 & -0.08 & -0.19 & 0.04 & \\
\hline & & & & & U.S. & 66 & $-0.14 * * *$ & -0.17 & -0.11 & \\
\hline \multirow{3}{*}{ Education } & \multirow{3}{*}{71} & \multirow{4}{*}{$-0.16^{* * *}$} & \multirow{4}{*}{-0.19} & \multirow{4}{*}{-0.13} & $\mathrm{COL}$ & 13 & $-0.17 * * *$ & -0.22 & -0.11 & \multirow{4}{*}{0.85} \\
\hline & & & & & IIND & 3 & -0.11 & -0.22 & 0.01 & \\
\hline & & & & & U.S. & 55 & $-0.16 * * *$ & -0.19 & -0.13 & \\
\hline \multirow{3}{*}{ Employment } & \multirow{3}{*}{37} & & & & $\mathrm{COL}$ & 13 & -0.03 & -0.11 & 0.05 & \\
\hline & & \multirow[t]{2}{*}{$-0.09 *$} & \multirow[t]{2}{*}{-0.16} & \multirow[t]{2}{*}{0.01} & IIND & 8 & -0.08 & -0.18 & 0.02 & \multirow[t]{2}{*}{4.29} \\
\hline & & & & & U.S. & 16 & $-0.14 * * *$ & -0.21 & -0.07 & \\
\hline \multirow{3}{*}{ Income } & \multirow{3}{*}{55} & \multirow{3}{*}{$-0.21 * * *$} & \multirow{3}{*}{-0.30} & \multirow{3}{*}{-0.12} & $\mathrm{COL}$ & 3 & -0.16 & -0.50 & 0.22 & \multirow{3}{*}{0.61} \\
\hline & & & & & IIND & 4 & -0.10 & -0.41 & 0.23 & \\
\hline & & & & & U.S. & 48 & $-0.23 * * *$ & -0.32 & -0.13 & \\
\hline
\end{tabular}

Individual Risk Markers

\begin{tabular}{|c|c|c|c|c|c|c|c|c|c|c|}
\hline \multirow{3}{*}{$\begin{array}{l}\text { Alcohol } \\
\text { Abuse }\end{array}$} & \multirow{3}{*}{163} & \multirow{3}{*}{$0.24 * * *$} & \multirow{3}{*}{0.19} & \multirow{3}{*}{0.28} & $\mathrm{COL}$ & 30 & $0.27 * * *$ & 0.19 & 0.35 & \multirow{3}{*}{3.24} \\
\hline & & & & & IIND & 15 & $0.18 * * *$ & 0.11 & 0.22 & \\
\hline & & & & & U.S. & 118 & $0.24 * * *$ & 0.22 & 0.27 & \\
\hline \multirow{3}{*}{ Drug Abuse } & \multirow{3}{*}{63} & \multirow{3}{*}{$0.26 * * *$} & \multirow{3}{*}{0.21} & \multirow{3}{*}{0.30} & $\mathrm{COL}$ & 5 & $0.32 * * *$ & 0.17 & 0.47 & \multirow{3}{*}{1.08} \\
\hline & & & & & IIND & 10 & $0.27 * * *$ & 0.16 & 0.38 & \\
\hline & & & & & U.S. & 48 & $0.25 * * *$ & 0.20 & 0.29 & \\
\hline
\end{tabular}

\section{Family of Origin Risk Markers}

\begin{tabular}{lcccccccccc} 
Child Abuse & \multirow{2}{*}{51} & $0.23 * * *$ & 0.19 & 0.26 & IIND & 6 & $0.25 * * *$ & 0.11 & 0.31 & 0.19 \\
in FOO & & & & & U.S. & 39 & $0.22 * * *$ & 0.18 & 0.27 & \\
& & & & & COL & 9 & $0.38 * * *$ & 0.30 & 0.46 & \\
Witnessing & \multirow{2}{*}{59} & \multirow{2}{*}{$0.27 * * *$} & \multirow{2}{*}{0.17} & 0.37 & IIND & 12 & $0.20 * * *$ & 0.12 & 0.28 & $11.82 * *$ \\
Parental IPV & & & & & U.S. & 38 & $0.24 * * *$ & 0.19 & 0.28 &
\end{tabular}

\section{Relationship Risk Markers}




\begin{tabular}{|c|c|c|c|c|c|c|c|c|c|c|}
\hline \multirow{3}{*}{ Control } & \multirow{3}{*}{30} & \multirow{3}{*}{$0.37 * * *$} & \multirow{3}{*}{0.28} & \multirow{3}{*}{0.44} & COL & 9 & $0.40^{* * *}$ & 0.26 & 0.53 & \multirow[b]{2}{*}{0.79} \\
\hline & & & & & IIND & 8 & $0.31 * *$ & 0.13 & 0.46 & \\
\hline & & & & & U.S. & 13 & $0.37 * * *$ & 0.25 & 0.49 & \\
\hline \multirow{3}{*}{$\begin{array}{l}\text { Emotional } \\
\text { Abuse }\end{array}$} & \multirow{3}{*}{97} & \multirow{3}{*}{$0.52^{* * * *}$} & \multirow{3}{*}{0.40} & \multirow{3}{*}{0.61} & $\mathrm{COL}$ & 5 & $0.63 * * *$ & 0.50 & 0.72 & \multirow{3}{*}{$10.06^{* *}$} \\
\hline & & & & & IIND & 15 & $0.40^{* * *}$ & 0.30 & 0.49 & \\
\hline & & & & & U.S. & 77 & $0.52 * * *$ & 0.49 & 0.56 & \\
\hline \multirow{3}{*}{$\begin{array}{l}\text { Relationship } \\
\text { Satisfaction }\end{array}$} & \multirow{3}{*}{72} & \multirow{3}{*}{$-0.22 * * *$} & \multirow{3}{*}{-0.31} & \multirow{3}{*}{-0.12} & $\mathrm{COL}$ & 4 & $-0.23^{* *}$ & -0.34 & -0.12 & \multirow{3}{*}{$8.55^{* *}$} \\
\hline & & & & & IIND & 8 & $-0.13 * * *$ & -0.22 & -0.03 & \\
\hline & & & & & U.S. & 60 & $-0.27 * * *$ & -0.30 & -0.24 & \\
\hline
\end{tabular}

Note. ${ }^{*} p<.05, * *, p<.01, * * * p<.001$. (two-tailed). COL. $=$ Collectivist. IIND. $=$ Individualistic. U.S = United States. Individualistic Countries (\# of studies): Australia (4), Canada (18), Holland (1), New Zealand (2), South Africa (5), and United States (210), Ukraine (1). Collectivist Countries (\# of studies): Albania (1), Bolivia (2), Brazil (1), Cambodia (1), ${ }^{\mathrm{a} C}$ Chile (1), China (1), Dominican Republic (1), ${ }^{\mathrm{a}}$ Eygpt (1) Ethiopia (2), , Hong Kong (4), ${ }^{\mathrm{a}}$ India (5), Indonesia (1), Iran (2), Iraq (1), Israel (2), Jordan (1), Kenya (1),, Mexico (1), Myanmar (1), ${ }^{b}$ Nicaragua (2), Nigeria (2), Pakistan (1), Palestine (1), ${ }^{b}$ Peru (2), ${ }^{a}$ Philippines (1) Rwanda (1), Singapore (1), Spain (1), Sri Lanka (1), Syria (1), Thailand (1), Turkey (3), and Uganda (1). ${ }^{\text {a }} 1$ study is from multiple samples in Jeyaseelan et. al., 2004. ${ }^{\mathrm{b}} 1$ study is from multiple samples in Flake \& Forste, 2006.

\section{International Individualist Countries versus United States.}

Our first two group comparison tested the magnitude of these eleven risk markers for male perpetrated IPV between the international individualist and U.S. groups (see Table 3). Nine of these risk markers did not significantly differ between these two groups, but two risk markers were significantly stronger for the U.S. group: emotional abuse $\left(Q^{b}(1)=7.02, p<.01\right)$ and relationship satisfaction $\left(Q^{b}(1)=8.80, p<.01\right)$. We found that for the U.S. group, emotional abuse $(r=.52, \mathrm{p}<.001)$ and low relationship satisfaction $(r=-.27, \mathrm{p}<.001)$ were significantly stronger risk markers for male perpetration of IPV in the U.S., compared to international individualist countries (emotional abuse: $r=.40, \mathrm{p}<.001$; and relationship satisfaction: $r=-.13$, $\mathrm{p}<.001$ ) Therefore, of the eleven risk markers examined, two (emotional abuse, and relationship satisfaction) were found to be significantly larger markers for the U.S. group compared to the individualist countries group. 
Table 3

Two Group Analysis of Risk markers for Male Perpetration of IPV: International Individualist Countries and the United States

\begin{tabular}{llllllllllll}
\hline & & \multicolumn{2}{c}{$95 \%$} & C.I. & & & & \multicolumn{2}{c}{$95 \%$ C.I. } & \\
Risk Marker & $k$ & Mean $r$ & $L L$ & $U L$ & Culture & $k$ & Mean $r$ & $L L$ & $U L$ & $Q^{\text {between }}$ \\
\hline
\end{tabular}

\section{Demographic Risk Markers}

\begin{tabular}{|c|c|c|c|c|c|c|c|c|c|c|}
\hline Age & 69 & $-0.14 * * *$ & -0.17 & -0.11 & $\begin{array}{l}\text { IIND } \\
\text { U.S. }\end{array}$ & $\begin{array}{c}3 \\
66\end{array}$ & $\begin{array}{l}-0.08 \\
-0.14 * * *\end{array}$ & $\begin{array}{l}-0.20 \\
-0.17\end{array}$ & $\begin{array}{r}0.05 \\
-0.11\end{array}$ & 0.96 \\
\hline Education & 58 & $-0.16^{* * *}$ & -0.19 & -0.12 & $\begin{array}{l}\text { IIND } \\
\text { U.S. }\end{array}$ & $\begin{array}{c}3 \\
55\end{array}$ & $\begin{array}{c}-0.11 \\
-0.16^{* * * *}\end{array}$ & $\begin{array}{l}-0.23 \\
-0.19\end{array}$ & $\begin{array}{r}0.01 \\
-0.13\end{array}$ & 0.67 \\
\hline Employment & 24 & $-0.12 * * *$ & -0.18 & -0.06 & $\begin{array}{l}\text { IIND } \\
\text { U.S. }\end{array}$ & $\begin{array}{c}8 \\
16\end{array}$ & $\begin{array}{l}-0.08 \\
-0.14 * * *\end{array}$ & $\begin{array}{l}-0.18 \\
-0.21\end{array}$ & $\begin{array}{r}0.02 \\
-0.07\end{array}$ & \\
\hline come & 52 & -0.21 & -0.30 & -0.12 & $\begin{array}{l}\text { IIND } \\
\text { U.S. }\end{array}$ & $\begin{array}{c}4 \\
48\end{array}$ & $\begin{array}{l}-0.10 \\
-0.22 * * *\end{array}$ & $\begin{array}{l}-0.41 \\
-0.32\end{array}$ & $\begin{array}{r}0.23 \\
-0.13\end{array}$ & \\
\hline
\end{tabular}

Individual Risk Markers

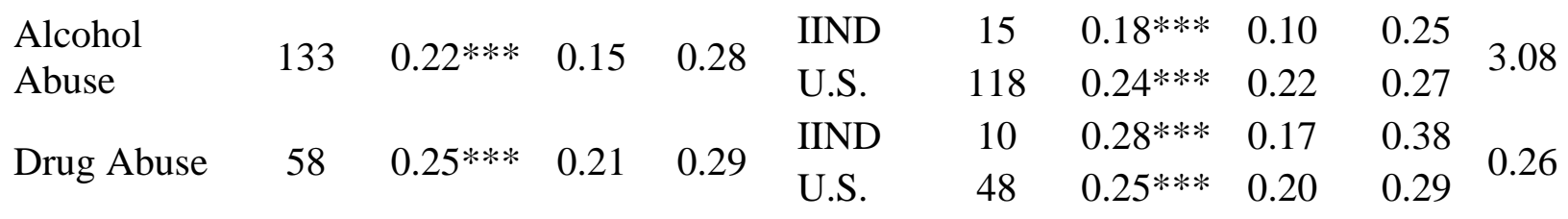

\section{Family of Origin Risk Markers}

\begin{tabular}{lllllllllll} 
Child Abuse & \multirow{2}{*}{45} & \multirow{2}{*}{$0.23 * * *$} & 0.19 & 0.27 & IIND & 6 & $0.25 * * *$ & 0.15 & 0.34 & 0.19 \\
in FOO & & & & & U.S. & 39 & $0.22 * * *$ & 0.18 & 0.26 & \\
Witnessing & \multirow{2}{*}{50} & \multirow{2}{*}{$0.23 * * *$} & 0.19 & 0.26 & IIND & 12 & $0.20 * * *$ & 0.13 & 0.27 & \\
Parental IPV & & & & & U.S. & 38 & $0.23 * * *$ & 0.19 & 0.28 & 0.61
\end{tabular}

\section{Relationship Risk Markers}

\begin{tabular}{|c|c|c|c|c|c|c|c|c|c|c|}
\hline \multirow{2}{*}{ Control } & \multirow{2}{*}{21} & \multirow{2}{*}{$0.35 * * *$} & \multirow{2}{*}{0.27} & \multirow{2}{*}{0.42} & IIND & 8 & $0.30^{* * *}$ & 0.16 & 0.42 & \multirow{2}{*}{0.89} \\
\hline & & & & & U.S. & 13 & $0.38 * * *$ & 0.28 & 0.46 & \\
\hline \multirow{2}{*}{$\begin{array}{l}\text { Emotional } \\
\text { Abuse }\end{array}$} & \multirow{2}{*}{92} & \multirow{2}{*}{$0.47 * * *$} & \multirow{2}{*}{0.34} & \multirow{2}{*}{0.58} & IIND & 15 & $0.40 * * *$ & 0.30 & 0.48 & \multirow{2}{*}{$7.02 * *$} \\
\hline & & & & & U.S. & 77 & $0.52 * * *$ & 0.49 & 0.55 & \\
\hline $\begin{array}{l}\text { elationship } \\
\text { atisfaction }\end{array}$ & 68 & $-0.21 *$ & -0.34 & -0.06 & $\begin{array}{l}\text { IIND } \\
\text { U.S. }\end{array}$ & $\begin{array}{c}8 \\
60\end{array}$ & $\begin{array}{l}-0.13 * * * \\
-0.27 * * * *\end{array}$ & $\begin{array}{l}-0.22 \\
-0.30\end{array}$ & $\begin{array}{l}-0.03 \\
-0.24\end{array}$ & $8.80 * *$ \\
\hline
\end{tabular}
Note. ${ }^{*} p<.05,{ }^{*}, p<.01,{ }^{* * *} p<.001$. (two-tailed). IIND $=$ Individualistic. U.S. $=$ 


\section{Collectivist Countries and the United States.}

Our second two group comparison tested the magnitude of the eleven risk markers for male perpetrated IPV between the collectivist countries and U.S. groups. Nine of these risk markers did not significantly differ between these two groups, but two risk markers were significantly different (see Table 4). Results indicated that young age was a significantly stronger risk marker $\left(Q^{b}(1)=5.65, p<.05\right)$ for male perpetration of IPV in the U.S. $(r=-.14, p<.001)$ compared to collectivist countries $(r=-.05, n s)$. In contrast, witnessing parental IPV was a significantly stronger risk marker $\left(Q^{b}(1)=7.45, p<.01\right)$ for male perpetration of IPV in collectivist countries $(r=.39, p<.001)$ compared to the U.S. $(r=.24, p<.001)$. Thus, young age was a stronger risk marker in the U.S. than in collectivist countries, whereas witnessing parental IPV was a stronger risk marker in collectivist countries than in the U.S.

Table 4

Two Group Analysis of Risk markers for Male Perpetration of IPV: Collectivist countries and the United States

\begin{tabular}{|c|c|c|c|c|c|c|c|c|c|c|}
\hline \multirow[b]{2}{*}{ Risk Marker } & \multirow[b]{2}{*}{$k$} & \multirow[b]{2}{*}{ Mean $r$} & \multicolumn{2}{|c|}{$95 \%$ C.I. } & \multirow[b]{2}{*}{ Culture } & \multirow[b]{2}{*}{$k$} & \multirow[b]{2}{*}{ Mean $r$} & \multicolumn{2}{|c|}{ 95\% C.I. } & \\
\hline & & & $L L$ & $U L$ & & & & $L L$ & $U L$ & $Q^{\text {between }}$ \\
\hline
\end{tabular}

\section{Demographic Risk Markers}

\begin{tabular}{|c|c|c|c|c|c|c|c|c|c|c|}
\hline Age & 76 & $-0.10^{*}$ & -0.19 & -0.01 & $\begin{array}{l}\text { COL } \\
\text { U.S. }\end{array}$ & $\begin{array}{l}10 \\
66\end{array}$ & $\begin{array}{l}-0.05 \\
-0.14 * * *\end{array}$ & $\begin{array}{l}-0.12 \\
-0.17\end{array}$ & $\begin{array}{l}-0.02 \\
-0.11\end{array}$ & $5.65 *$ \\
\hline Education & 68 & $-0.16 * * *$ & -0.19 & -0.13 & $\begin{array}{l}\text { COL } \\
\text { U.S. }\end{array}$ & $\begin{array}{l}13 \\
55\end{array}$ & $\begin{array}{l}-0.17 * * * \\
-0.16 * * *\end{array}$ & $\begin{array}{l}-0.22 \\
-0.19\end{array}$ & $\begin{array}{l}-0.11 \\
-0.13\end{array}$ & 0.06 \\
\hline Employment & 29 & -0.09 & -0.19 & 0.02 & $\begin{array}{l}\text { COL } \\
\text { U.S. }\end{array}$ & $\begin{array}{l}13 \\
16\end{array}$ & $\begin{array}{l}-0.03 \\
-0.14 * * *\end{array}$ & $\begin{array}{l}-0.12 \\
-0.22\end{array}$ & $\begin{array}{r}0.05 \\
-0.07\end{array}$ & 3.55 \\
\hline Income & 51 & $-0.22 * * *$ & -0.31 & -0.13 & $\begin{array}{l}\text { COL } \\
\text { U.S. }\end{array}$ & $\begin{array}{c}3 \\
48\end{array}$ & $\begin{array}{l}-0.15 \\
-0.23 * * *\end{array}$ & $\begin{array}{l}-0.52 \\
-0.32\end{array}$ & $\begin{array}{r}0.24 \\
-0.13\end{array}$ & 0.09 \\
\hline \multicolumn{11}{|c|}{ Individual Risk Markers } \\
\hline $\begin{array}{l}\text { Alcohol } \\
\text { Abuse }\end{array}$ & 148 & $0.25 * * *$ & 0.22 & 0.27 & $\begin{array}{l}\text { COL } \\
\text { U.S. }\end{array}$ & $\begin{array}{c}30 \\
118\end{array}$ & $\begin{array}{l}0.26 * * * \\
0.24 * * *\end{array}$ & $\begin{array}{l}0.21 \\
0.22\end{array}$ & $\begin{array}{l}0.32 \\
0.27\end{array}$ & 0.43 \\
\hline
\end{tabular}




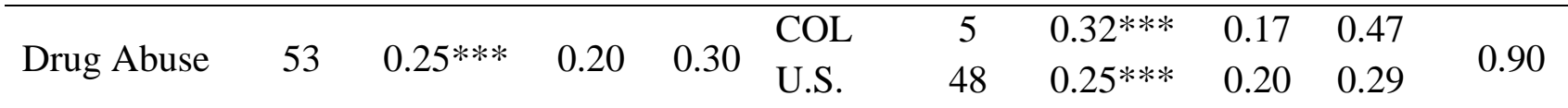

\section{Family of Origin Risk Markers}

Child Abuse

in FOO

$45 \quad 0.22 * * *$

$0.18 \quad 0.27$

$\begin{array}{lll}\text { COL } & 6 & 0.22 * * *\end{array}$

$0.11 \quad 0.32$

U.S. $\quad 390.23 * * *$

$0.18 \quad 0.27$

0.02

Witnessing

Parental IPV

$47 \quad 0.31 * * * \quad 0.16 \quad 0.44$

$\begin{array}{lcc}\text { COL } & 9 & 0.39 * * * \\ \text { US } & 38 & 0.24 * * *\end{array}$

$\begin{array}{ll}0.30 & 0.47\end{array}$

$0.19 \quad 0.29$

\section{Relationship Risk Markers}

\begin{tabular}{|c|c|c|c|c|c|c|c|c|c|c|}
\hline Control & 22 & $0.38 * * *$ & 0.28 & 0.48 & $\begin{array}{l}\text { COL } \\
\text { U.S. }\end{array}$ & $\begin{array}{c}9 \\
13\end{array}$ & $\begin{array}{l}0.40 * * * \\
0.37 * * *\end{array}$ & $\begin{array}{l}0.24 \\
0.23\end{array}$ & $\begin{array}{l}0.54 \\
0.50\end{array}$ & 0.09 \\
\hline $\begin{array}{l}\text { Emotional } \\
\text { Abuse }\end{array}$ & 82 & $0.56^{* * *}$ & 0.45 & 0.65 & $\begin{array}{l}\text { COL } \\
\text { U.S. }\end{array}$ & $\begin{array}{c}5 \\
77\end{array}$ & $\begin{array}{l}0.62 * * * \\
0.52 * * *\end{array}$ & $\begin{array}{l}0.50 \\
0.49\end{array}$ & $\begin{array}{l}0.73 \\
0.56\end{array}$ & 2.42 \\
\hline $\begin{array}{l}\text { Relationship } \\
\text { Satisfaction }\end{array}$ & 64 & $-0.27 * * *$ & -0.30 & -0.24 & $\begin{array}{l}\text { COL } \\
\text { U.S. }\end{array}$ & $\begin{array}{c}4 \\
60\end{array}$ & $\begin{array}{l}-0.23 * * * \\
-0.27 * * *\end{array}$ & $\begin{array}{l}-0.34 \\
-0.30\end{array}$ & $\begin{array}{l}-0.12 \\
-0.24\end{array}$ & 0.45 \\
\hline
\end{tabular}

\section{International Individualist and Collectivist Countries.}

Our final two group comparison tested the magnitude of the eleven risk markers for male perpetrated IPV between the international individualist and collectivist groups. Nine of the risk markers did not significantly differ between these two groups, but two risk markers were significantly different (see Table 5). The results of this two group analysis revealed that for collectivist countries, witnessing parental IPV $(r=.38, p<.001)$ and emotional abuse $(r=.62, p$ $<.001)$, were significantly stronger risk markers $\left(Q^{b}(1)=10.98, p<.001\right.$ and $Q^{b}(1)=8.09, p$ $<.01$ respectively) for male perpetration of IPV, compared to international individualist countries (witnessing parental IPV: $r=.20, p<.001$ and emotional abuse: $r=.40, p<.001$ ). Thus, two risk markers, witnessing parental IPV and emotional abuse, were stronger risk markers in collectivist countries than in international individualist countries.

Table 5

Two Group Analysis of Risk markers for Male Perpetration of IPV: International Individualist and Collectivist countries 


\begin{tabular}{|c|c|c|c|c|c|c|c|c|c|c|}
\hline \multirow[b]{2}{*}{ Risk Marker } & \multirow[b]{2}{*}{$k$} & \multirow[b]{2}{*}{ Mean $r$} & \multicolumn{2}{|c|}{ 95\% C.I } & \multirow[b]{2}{*}{ Culture } & \multirow[b]{2}{*}{$k$} & \multirow[b]{2}{*}{ Mean $r$} & \multicolumn{2}{|c|}{ 95\% C.I. } & \\
\hline & & & $L L$ & $U L$ & & & & $L L$ & $U L$ & $Q^{\text {between }}$ \\
\hline
\end{tabular}

\section{Demographic Risk Markers}

\begin{tabular}{|c|c|c|c|c|c|c|c|c|c|c|}
\hline \multirow{2}{*}{ Age } & \multirow{2}{*}{13} & \multirow{2}{*}{$-0.06^{*}$} & \multirow[t]{2}{*}{-0.10} & \multirow{2}{*}{-0.02} & $\mathrm{COL}$ & 10 & $-0.05^{*}$ & \multirow{2}{*}{$\begin{array}{l}-0.10 \\
-0.15\end{array}$} & \multirow{2}{*}{$\begin{array}{c}0.003 \\
0.01\end{array}$} & \multirow[t]{2}{*}{0.1} \\
\hline & & & & & IIND & 3 & -0.07 & & & \\
\hline \multirow{2}{*}{ Education } & \multirow{2}{*}{13} & \multirow{2}{*}{$-0.15 * * *$} & \multirow{2}{*}{-0.22} & \multirow{2}{*}{-0.11} & $\mathrm{COL}$ & 13 & $-0.16^{* * *}$ & -0.22 & -0.11 & \\
\hline & & & & & IIND & 3 & $-0.11^{*}$ & -0.21 & 0.004 & \\
\hline \multirow{2}{*}{ Employment } & \multirow{2}{*}{21} & \multirow{2}{*}{-0.05} & \multirow{2}{*}{-0.10} & \multirow{2}{*}{0.01} & COL & 13 & -0.03 & -0.10 & 0.04 & \\
\hline & & & & & IIND & 8 & $-0.07 *$ & -0.16 & 0.01 & \\
\hline \multirow{2}{*}{ Income } & \multirow{2}{*}{7} & \multirow{2}{*}{$-0.13 *$} & \multirow{2}{*}{-0.24} & \multirow{2}{*}{-0.01} & $\mathrm{COL}$ & 3 & -0.17 & -0.34 & 0.01 & \\
\hline & & & & & IIND & 4 & -0.10 & -0.24 & 0.05 & \\
\hline
\end{tabular}

\section{Individual Risk Markers}

\begin{tabular}{lcllllllll} 
Alcohol Abuse & 45 & $0.23 * * *$ & 0.14 & 0.31 & COL & 30 & $0.27 * * *$ & 0.20 & 0.33 \\
& & & & & IIND & 15 & $0.18 * * *$ & 0.08 & 0.27 \\
Drug Abuse & 15 & \multirow{2}{*}{$0.29 * * *$} & 0.20 & 0.38 & COL & 5 & $0.32 * * *$ & 0.16 & 0.47 \\
& & & & & IIND & 10 & $0.28 * * *$ & 0.16 & 0.38
\end{tabular}

\section{Family of Origin Risk Markers}

Child Abuse in

FOO

$120.23 * * *$

Witnessing

Parental IPV $\begin{array}{ll}0.15 & 0.31\end{array}$

COL

COL

IIND
0.110 .46

Relationship Risk Markers

Control

$170.36^{* * * *}$

$0.25 \quad 0.46$

Emotional

Abuse

$$
20 \quad 0.52 * * *
$$

$0.26 \quad 0.70$

Relationship

Satisfaction
$12-0.17 * * \quad-0.27 \quad-0.07$

COL

$\mathrm{COL}=5 \quad 0.63^{* * *}$

$\begin{array}{lll}\text { COL } 5 & 5.63 * * *\end{array}$

IIND $15 \quad 0.40 * * *$

COL $4 \quad-0.23 * * *$

IIND

$\begin{array}{ll}6 & 0.22 * * * \\ 6 & 0.25 * * *\end{array}$

0.11

0.13

0.32

0.35

0.76

0.30

0.13

0.46

0.27

$10.98^{*}$

**

Note. ${ }^{*} p<.05,{ }^{* *}, p<.01,{ }^{* * *} p<.001$. (two-tailed). COL $=$ Collectivist. IIND. $=$ Individualist.

\section{Discussion}

Although a great deal of research on IPV has been conducted within the U.S. and North America, there is a need for more research on risk markers for IPV worldwide. The primary goal of the present study was to contribute to the literature by using a meta-analytic approach to 
examine risk markers of IPV in different cultural contexts to identify possible similarities and differences. Based on Hofstede's Dimensional Model of Culture (Hofstede, 2011), which categorizes countries as 'individualist' or 'collectivist', we conducted a series of four analyses to examine the strength of risk markers for IPV worldwide, considering the possibility of cultural influences of collectivist/individualist ideals on male-to-female physical IPV perpetration. Hofstede's characterization of individualist and collectivist cultures provides a theoretical basis for many of the differences found between groups.

\section{Demographic Risk Markers}

Overall, the demographic risk marker we examined—age, education, employment, and income-had small association with men being IPV perpetrators. We hypothesized that because family plays a more active role in collectivist countries than in individualist countries, the risk markers of age, education, income, and employment status may have a weaker relationship with IPV in collectivist countries than in individualist countries. Results indicated that younger males were at greater risk for perpetrating IPV in the U.S. compared to collectivist countries but not individualist countries. Interestingly, the international individualist and U.S. groups had a number of differences in which demographic risk markers were related to male perpetration of physical IPV with low education emerging as a significant risk marker for the U.S. but not the international individualist group. These results for age lend partial support for our hypothesis.

As expected based on Hofstede's (2011) depiction of individualist societies as emphasizing "I" consciousness, autonomy, pleasure seeking, and financial security, demographic factors (in the U.S. and other individualist countries) seem to be stronger IPV risk markers than in collectivist countries since being older, more educated, and having money come with more status and power in individualist societies. Not having these characteristics may lead a person to 
seek other forms of power which could include violence. In collectivist cultures there tends to be a sense of family solidarity which may provide support for their young, low earning, and undereducated men, which may explain why each of the demographic risk markers, except for age, were not significant risk markers in collectivist countries. While younger men in collectivist cultures may have more familial and social support than in more individualist cultures, the patriarchal structure across most cultures provides a narrow view of how, and if, men should use violence as a conflict tactic. Younger men tend to be more susceptible to these culturally supported norms.

\section{Individual Risk Markers}

Overall, the individual risk markers - drug abuse and alcohol problems - examined in this study had small-to-moderate relationships with IPV in all three groups. In addition, they were significant risk markers for IPV in all three groups. However, no significant differences were found for the strength of alcohol problems or drug use as predictors of IPV between the cultures in the three group or two group analyses. A lack of difference between groups, but a consistent association between these risk markers and IPV perpetration, suggests that alcohol problems and drug use are important risk markers for IPV regardless of cultural grouping and that targeting these risk markers through intervention and prevention efforts is crucial.

\section{Family of Origin Risk Markers}

Both witnessing parental IPV and experiencing child abuse were significantly associated with increased risk for IPV perpetration across all three groups. Overall, the effect sizes for the family of origin risk markers were small-to-moderate in size (see Table 3). Based on our findings, growing up in a violent home appears to be an important risk marker across all cultures with some differences between individualist and collectivist countries. 
Our hypothesis was partially supported with witnessing parental IPV being a stronger risk marker for male perpetration in collectivist countries compared to the U.S. and individualist countries (refer to Tables 4 and 5). However, there were not significant differences between individualist and collectivist countries for child abuse as a risk marker. Considering the "we" consciousness, value of loyalty, and desire to maintain harmony, IPV may be used to enforce loyalty and congruence in relationships as well as prevent shame in collectivist societies. IPV prevention programs need to assess for a history of witnessing parental IPV or being abused as a child regardless of culture but it may be especially pertinent to examine a history of witnessing parental IPV more closely in collectivist societies.

\section{Relationship Risk Markers}

All relationship risk markers examined in this study (emotional abuse, control, and relationship satisfaction) were significantly related to increased risk for IPV perpetration across all three groups, with emotional abuse having the largest overall effect out of all the risk markers in the study, followed by control. Overall, these risk markers ranged from small-to-large in size and suggest cultural differences across these three groups (see Table 2). Perpetrating emotional abuse was a significantly stronger risk marker for males' perpetration of IPV for both the collectivist and U.S. groups compared to the individualist group. Low relationship satisfaction was a stronger risk marker for males perpetrating physical IPV in the U.S. compared to individualist countries (Table 3), but not compared to collectivist countries. Although we hypothesized that power and control would be a stronger risk marker in collectivist countries than in individualist countries because of the stronger influence of patriarchy, we hypothesized that relationship satisfaction may be more strongly related to IPV in individualist countries than 
in collectivist countries because of the tendency towards individual choice in mate selection in individualist countries..

Our hypotheses were only partially supported. Emotional abuse, control, and low relationship satisfaction were not significantly different risk markers when studies conducted in the U.S. and collectivist countries were compared in the two group analysis (Table 4). However, when studies conducted in collectivist and individualist countries were compared, the strength of emotional abuse as a risk marker significantly differed but the impact of low relationship satisfaction and control did not significantly differ (Table 5).

Implications of these cultural differences can provide helpful directions for clinicians and IPV researchers. First, emotional abuse needs be addressed as an important risk marker as demonstrated by its strong association with physical IPV in all three groups. The larger effect size found in U.S. and collectivist countries compared to international individualist countries points to cultural differences that make this a powerful risk marker in the U.S. and collectivist countries. Given the strong empirical evidence of emotional abuse as a risk marker for physical IPV and the association of low relationship satisfaction with physical IPV, these findings are not unexpected. However, in both cases, the collectivist group had larger effect sizes than the U.S. and individualist group. Emotional dependence is a characteristic of Hofstede's definition of collectivism (Brewer \& Chen, 2007, p.134). When a person is emotionally dependent, the real or imagined fear of a partner leaving has been found to lead to increased violence in male perpetrators of physical IPV (Roberts \& Noller, 1998). Both emotional abuse and low relationship satisfaction may signal emotional disengagement, a lack of solidarity and therefore a lack of belonging, harmony, and "we" consciousness which may lead to use of violence to maintain these standards in a collectivist society. 


\section{Comparison of Findings from U.S. versus Other Groups}

An important overall finding in this study is that risk markers for IPV vary across cultural groups, with unexpectedly greater similarities in effects between the U.S. and collectivist countries than between the U.S. and other individualist countries. We offer several potential explanations for why two risk markers (i.e., emotional abuse, relationship satisfaction) are stronger risk markers of male IPV perpetration in the U.S. than in other individualist countries, and why young age has a stronger association with IPV perpetration in the U.S. than in collectivist countries.

One potential explanation is that on Hofstede's scale, the U.S. has the highest individualism score (91). In theory, countries that have a higher individualism scores hold the characteristics described by Hofstede in higher esteem than those with a lower score. Individualist countries place more importance taking care of the self and immediate family (Hofstede, 2011) which puts a higher value on the few close interpersonal relationships that one does have. Thus, it is possible that emotional abuse is an attempt to maintain the close relationship and low relationship satisfaction signals the impending loss of a close relationship which creates a context where violence may occur. However, differences between the U.S. and the other groups with regards to the strength of young age as a risk marker is less easily conceptualized using Hofstede's theory.

A second possible explanation for the similarities been the U.S. and collectivist countries with regards to the strength of various risk markers for IPV may stem from factors such as higher income inequality or lower access to free health care in the U. S. versus other individualist countries (especially Canada which contributed the highest number of effect sizes to this study next to the U.S.) UNICEF's annual "State of the World's Children Reports" (cited in Collison, 
Dey, Hannah, \& Stevenson, 2007) indicated that the U.S. consistently rates as the highest in income inequality and child mortality rates among wealthier countries. Income inequality has frequently been demonstrated to be a determinant of population health (Ram, 2006). Ram suggests that "there is some support for the proposition that while income may be relatively more important for health in less developed countries, the role of income inequality may be stronger in developed countries" (p. 779). Future research might use level of income inequality as a grouping factor rather than collectivist versus individualistic rating.

Another possible explanation for the finding that effect sizes for the relationship between IPV risk markers in the U.S. are more similar to those in collectivist countries may have to do with the availability of mental health care in the U.S. versus other individualist countries. A study by Vasiliadis and colleagues (2007) compared the prevalence of depression and determinants of mental health care use in U.S. versus Canada and found that although there was no difference in the prevalence of depression and mental health care use between Canada and U.S., disparities in treatment seeking were found to be associated with lack of medical insurance in the United States. An important finding from the present study is that younger men with lower relationship satisfaction, and who were emotionally abusive, were at greater risk for perpetrating IPV in the U.S. than in other cultures. In addition, low income was a significant risk factor in the U.S., but not in collectivist or individualistic international countries. It is possible that similarity between effect sizes in the U.S. and in collectivist countries may result from differences between the U.S. and other individualist countries in terms of income disparity or availability of free mental health care.

\section{Limitations}


"Societal cultures reside in (often unconscious) values, in the sense of broad tendencies to prefer certain states of affairs over others" (Hofstede, 2001, p.55). Because individual differences (i.e. preferences and personalities) exist within a family, family differences (i.e. dynamics and expectations) within cultures, cultural differences (i.e. values and ideals) within regions or cities, and regional differences (i.e. resources and infrastructure) within countries, any "categorical distinction" will experience heterogeneity and exceptions. Because Hofstede’s cultural dimensions are only calculated at the country level, we were unable to examine individualist and collective values within countries. However, were this possible, we still would have been limited by the fact that even within regions, states, and cities, different groups have differing individual and collective values (Oyserman, Coon, \& Kemmelmeier, 2002). Thus, to truly understand the factors that make individuals from one country differ culturally from individuals in another country, numerous dimensions and multiple levels of culture need consideration (Hofstede, 2010). This provides a challenge when assessing for IPV risk markers on such a broad level.

Another limitation of our study was its exclusion of studies not available in English. It is likely that there are articles written in other languages that we were not able to include because they were not available in English. As a result there were many over representations and under representations of countries within each cultural dimension. For example, Canada accounted for the majority of the studies in international individualist countries as did China for the collectivist countries.

Meta-analyses are limited by the quality and availability of the studies that they analyze. It should also be noted that these results are correlational not causal. It is also possible that the low number of effect sizes for some risk markers in the individualistic and collectivist group (e.g education) may explain some non-significant findings. For this study there were two additional 
limitations that warrant further investigations. While there were some consistent measures used for each risk marker, there were also many differences which may warrant further investigation about how these risk markers are measured across cultures. For example, future researchers could examine relationship factors, and how each study measured these factors more carefully to determine if differences in strengths of risk markers vary according the measure used. The focus of this meta-analysis was to investigate risk markers across cultures rather than compare the ways that IPV are measured in each study. Although we did not find differences across studies regarding whether clinical or community samples were included, future research could also address more clearly differences in strength of risk markers according to prevalence or severity of violence.

Finally, this study considered IPV as a unitary phenomenon although earlier research has made it clear that there are subtypes of violence. Few studies made use of Johnson's (1995; 2001) typology describing differences in IPV (e.g., situational violence versus intimate terrorism). Therefore, this study is limited since it did not examine differences between the strengths of the risk markers based on type of violence, although we did assess differences based on community versus clinical samples and found no significant differences.

\section{Future Research Directions}

The studies included in the meta-analysis were limited to male perpetration. There are few studies on female perpetration, female victimization, and male victimization outside of the U.S. and North America. When looking at IPV through a cultural lens, studying both male and female IPV would help to better understand how IPV might be different in other cultures. Thus, more studies are needed in these areas. Furthermore, we were unable to include many potential risk markers in our analyses because there were not enough effect sizes (minimum of three) in 
the groups - typically collectivist countries. While there may be many aspects of IPV that we understand from research in the U.S. there is a risk that treatment for IPV can become blind to cultural differences if the field of IPV is not further informed by research from within various cultures. More research from other areas around the world will further elucidate the how risk markers differ in the context of diverse cultural values and practices.

\section{Conclusion}

Overall, our findings suggest that risk markers for IPV vary across cultural groups, with unexpectedly greater similarities in effects between the U.S. and collectivist countries than between the U.S. and individualist countries. This information provides a number of insights, the most prominent being that understanding IPV in a cultural context is necessary when determining markers associated with risk for perpetration and defining appropriate foci for intervention in a particular culture.

\section{Acknowledgments}

This research is based on work supported by the US Air Force Division of Behavioral Health and the US Department of Agriculture, National Institute of Food and Agriculture. 


\section{In-text References}

Author, et. al. (in press)

Author et. al. (2015).

Author et. al. (2008).

Author et. al. (2004).

Author et. al. (2000).

Borenstein, M., Hedges, L., Higgins, J., \& Rothsteine, H. (2005) Comprehensive Meta-Analysis (Version 2) [Computer software \}. Englewood, NJ: Biostat.

Brewer, M. B., \& Chen, Y. R. (2007). Where (who) are collectives in collectivism? Toward conceptual clarification of individualism and collectivism. Psychological review, 114(1), 133.

Burrus, K. J., \& Cobb, R. J. (2011). Spouses' perceptions of aggression and associations with relationship satisfaction. Partner Abuse, 2(2), 189-207.

Capaldi, D. M., Knoble, N. B., Shortt, J. W., \& Kim, H. K. (2012). A systematic review of risk factors for intimate partner violence. Partner Abuse, 3(2), 1-194.

Card, N. A. (2012). Applied meta-analysis for social science research. New York, NY: Guilford Press.

Chan, K. L. (2004). Correlates of wife assault in Hong Kong Chinese families. Violence and Victims, 19(2), 189-201. doi:10.1891/vivi.19.2.189.64104 
Collison, D., Dey, C., Hannah, G., \& Stevenson, L. (2007). Income inequality and child mortality in wealthy nations, Journal of Public Health, 29, 114-117. 10.1093/pubmed/fdm009 |A

Eisikovits, Z., Winstok, Z., \& Fishman, G. (2004). The first Israeli national survey on domestic violence. Violence Against Women, 10(7), 729-748.

Esquivel-Santoveña, E. E., Lambert, T. L., \& Hamel, J. (2013). Partner abuse worldwide. Partner Abuse, 4(1), 6-75. doi:http://dx.doi.org/10.1891/1946-6560.4.1.6

Garcia-Moreno, C., Pallitto, C., Devries, K., Stockl, H., Watts, C., \& Abrahams, N. (2013). Global and regional estimates of violence against women: prevalence and health effects of intimate partner violence and non-partner sexual violence. World Health Organization, 2013, 1-50.

Hadas, D., Markovitzky, G., \& Sarid, M. (2008). Spousal violence among immigrants from the Former Soviet Union-General population and welfare recipients. Journal of Family Violence, 23(7), 549-555.

Hawkins, A. J., Blanchard, V. L., Baldwin, S. A., \& Fawcett, E. B. (2008). Does marriage and relationship education work? A meta-analytic study. Journal of Consulting and Clinical Psychology, 76(5), 723-734. doi: http://dx.doi.org/10.1037/a0012584

Hofstede, G. (1984). Culture's consequences: International differences in work-related values. Sage series on cross-cultural research and methods (Vol. 5). CA: Sage Publications.

Hofstede, G. (2001) Culture's Consequences: Comparing Values, Behaviors, Institutions, and Organizations across Nations. Second Edition, Thousand Oaks CA: Sage Publications. 
Hofstede, G., Hofstede, G.J., Minkov, M., (2010). Cultures and organizations: Software of the mind. (3rd Ed). NY: McGraw-Hill

Hofstede. (2011). Dimensionalizing Cultures: The Hofstede Model in Context. Online Reading in Psychology and Culture, Unit 2.

Hunter, J. \& Schmidt, F. (2004). Methods of meta-analysis: Correcting error and bias in research findings (2nd ed.). Thousand Oaks, CA: Sage.

Jeyaseelan, L., Sadowski, L. S., Kumar, S., Hassan, F., Ramiro, L., \& Vizcarra, B. (2004). World studies of abuse in the family environment-risk factors for physical intimate partner violence. Injury Control and Safety Promotion, 11(2), 117-124.

Johnson, M. P., Leone, J. M., \& Xu, Y. (2014). Intimate Terrorism and Situational Couple Violence in General Surveys Ex-Spouses Required. Violence against women, 20(2), 186207.

Jose, A. (2010). Risk factors for physical aggression in couples (Doctoral dissertation). ProQuest Dissertations and Theses. (3179128)

Koenig, M. A., Saifuddin, A., Mian, B. H., \& A B M Khorshed Alam,Mozumder. (2003).

Women's status and domestic violence in rural Bangladesh: Individual- and communitylevel effects. Demography (Pre-2011), 40(2), 269-88.

Krahé, B., Bieneck, S., \& Möller, I. (2005). Understanding gender and intimate partner violence from an international perspective. Sex Roles, 52(11-12), 807-827. doi:http://dx.doi.org/10.1007/s11199-005-4201-0 
Langhinrichsen-rohling, J. (2010). Controversies involving gender and intimate partner violence in the United States. Sex Roles, 62(3-4), 179-193. doi:http://dx.doi.org/10.1007/s11199009-9628-2

O’Leary, K. D., Tintle, N., Bromet, E. J., \& Gluzman, S. F. (2008). Descriptive epidemiology of intimate partner aggression in Ukraine. Social psychiatry and psychiatric epidemiology, 43(8), 619-626.

O'Leary, K. D. (1999). Psychological abuse: A variable deserving critical attention in domestic violence. Violence and Victims, 14(1), 3-23. Retrieved from http://search.proquest.com.er.lib.k-state.edu/docview/208555852?accountid=11789

Orwin, R. G. (1983). A fail-safe N for effect size in meta-analysis. Journal of educational statistics, 157-159.

Oyserman, D., Coon, H. M., \& Kemmelmeier, M. (2002). Rethinking individualism and collectivism: evaluation of theoretical assumptions and meta-analyses. Psychological bulletin, 128(1), 3-72.

Ram, R. (2006). Further examination of the cross-country association between income inequality and population health. Social Science \& Medicine, 62, 799-791. http://dx.doi.org/10.1016/j.socscimed.2005.06.034

Roberts, N., \& Noller, P. (1998). Attachment theory and close relationships, edited by Simpson, J. A., Rholes, W. S., 317-350. New York, NY, Guilford Press.

Rosenthal, R. (1979). The file drawer problem and tolerance for null results. Psychological Bulletin, 86(3), 638-641. 
Vasiliadis, H. M., Lesage, A., Adair, C., Wang, P. S., \& Kessler, R. C. (2007). Do Canada and the United States differ in prevalence of depression and utilization of services?. Psychiatric Services, 58(1), 63-71.

White, H. R., \& Chen, P. H. (2002). Problem drinking and intimate partner violence. Journal of Studies on Alcohol and Drugs, 63(2), 205-214.

Yoshioka, M. R., \& Choi, D. Y. (2005). Culture and interpersonal violence research paradigm shift to create a full continuum of domestic violence services. Journal of interpersonal violence, 20(4), 513-519. 